may infer that there is something more than the mere valvular lesion. The ancillary factor may not always be adherent pericardium; it may be a fibroid myocarditis, but it is often the former.

4. In the cases of so-called quiet ascites, chiefly occurring in young subjects, in which cirrhosis of the liver and tuberculous peritonitis can be excluded. I have reference to the so-called pericarditic pseudocirrhosis, a subject ably discussed before this society by Dr. Kelly.

Since the beginning of the year to the present time, 9 cases of acute pericarditis have come under my observation: 2 I saw through the courtesy of my colleague, Dr. Thomas G. Ashton, at the Philadelphia Hospital; 2 occurred in my own wards in that institution; 3 were observed during my term of service at the Polyclinic Hospital; 1 was in a patient of Dr. Francis T. Stewart, and $1 \mathrm{I}$ saw in consultation with Dr. L. Podolski. Of these 9 cases 3 were in the female and 6 in the male sex; 4 of the patients were colored persons; 3 were tapped -1 with a negative result.

Etiologically the cases may be classified as follows: Probably of rheumatic origin, 4; associated with pneumonia and nephritis, 1 ; secondary to operation for the radical cure of hernia, 1 ; secondary to gallstone colic, 1 ; of unknown origin, 2 . Of the 9 cases, 7 were associated with endocarditis and 2 were unaccompanied with any evidence of endocardial lesion; 2 ended fatally, the autopsy showing fibrinous pericarditis with adhesions.

I have confined myself to a mention of these few details in regard to the cases so as not to lengthen the paper unduly. Those interested in the subject will find these cases more fully discussed in the forthcoming volume of the Transactions of the College of Physicians of Philadelphia, 1904, vol. xxvi.

\title{
TWO CASES OF PARACENTESIS OF THE PERICARDIUM. ${ }^{1}$
}

\author{
By JoHN B. RoBerts, M.D., \\ PROFESSOR OF BURGERT IN THE PHILADELPHIA POLYCLINIC.
}

THE glory of modern surgery can be appreciated only by practitioners who, in their youth, were familiar with the almost universal occurrence of operative sepsis. Surgical timidity, due to "inflammatory fever" and its results, made the experienced surgeons of our youthful days skeptical of the opinions of those of us who saw the coming of the new era.

The contention that trephining was not a serious procedure and ought to be adopted in nearly all fractures of the cranial vault ${ }^{2}$

\footnotetext{
1 Read before the College of Physicians of Philadelphia, May 4, 1904.

2 Roberts, Operative Surgery of Human Brain, Transactions of the American Surgical Association, 1885 .
} 
aroused opposition, and the advocacy of suprapubic cystotomy in preference to perineal incision was regarded as foolishness. ${ }^{1}$

So it was with my proposition that the diseased pcricardium should be treated as the pleura in similar pathological conditions. That the pericardium should be promptly aspirated (1876), if it contained a serous or hemorrhagic effusion unaffected by the administration of drugs, and that it be kept empty by incision and drainage (1876), if the fluid contents were purulent, ${ }^{2}$ was doubted. These procedures are now routine operations. It then seemed hazardous to incise the chest wall and pericardium to clear up a doubtful diagnosis of dilated heart or pericardial effusion (1881). ${ }^{3}$ The advocacy of heart suture as a therapeutic procedure for wounds of the cardiac wall (1881 and 1883) seemed in 1882 to be a youthful indiscretion, if one could judge from the tone of the Fellows of this College." A formal method of attacking the pericardium and heart by a trap-door resection of the costal cartilages, devised in 1897,5 was, however, the outcome of these studies.

This obstructive conservatism is, however, the usual attitude of mind of the middle-aged, and surgeons of that period of life need to bear in mind that most of what the profession learns is taught by young men.

To-night scveral cases of pericardicentesis and of pericardial drainage have been reported, and two Fellows, Dr. John H. Gibbon and Dr. Francis T. Stewart, have each added to surgical literature an instance of heart suture for penetrating wound. In Dr. Stewart's case the patient recovered. I feel honored that both are ex-resident physicians of the Polyclinic Hospital.

'Through the courtesy of Dr. David Riesman I recently had the opportunity of aspirating the pericardium in two patients with pericarditis, and desire to join him in the record of the cases. Both were tapped with the pericardial aspirating trocar devised and described in 1877 and 1881.

Roberts' perieardial aspirating trocar, consisting of a hollow needle and an internal cannula with flexible end.

CAsE I.-The patient, a colored woman, had been admitted to the Polyclinic Hospital the day before with pericarditis and bronchopneumonia.

Tapping was done March 9, 1904, at 6 P.M., in the fourth left

1 Roberts, Transactions of the American Surgical Association, 1884, p. 169.

2 New York Medical Journal, December, 1876.

${ }^{3}$ Annals of Anatomy and Surgery, December, 1881, p. 250; Medical News, January 13, 1883.

4 Transactions of the College of Physicians of Philadelphia, 1883.

5 The American Journal of the Medral Sciences, December, 1897. 
interspace over the apex beat, which could be distinctly felt. The point of puncture was about three and one-half inches to the left of the middle line of the sternum, under the left mammary gland, and perhaps through its lower edge. The Roberts pericardial trocar was employed, and worked satisfactorily, the vacuum of the aspirating pump being attached after a little fluid had flowed from the trocar. The introduction of the needle, which was made without attaching the aspirator, was followed by pushing forward the internal cannula. The trocar was introduced to a depth of about $3 \frac{1}{2} \mathrm{~cm}$. The point gave the impression of being within a cavity, and the beat of the heart against it was easily felt. The external portion of the trocar oscillated with the cardiac pulsations. Three ounces of a very bloody serum were drawn off. That this was not pure blood was shown by the lightish color and the yellowish-red stain made on the towels when some drops soiled them. In order to make a strong vacuum, after the small bottle was about half-filled, the aspirating apparatus was detached. The contents of the bottle had coagulated so promptly that it took a little time to wash out the bottle and to obtain some of the fluid in sterile test-tubes for bacteriological examination. The aspirator was attached to the trocar again, but no further fluid was obtained. When the aspirating apparatus was detached there was a rush of air into the bottle, showing that a vacuum had been properly obtained. No air flowed into the chest, as the trocar on being withdrawn was found to be plugged with a clot of bloody lymph.

The wound was sealed with collodion and cotton. I thought it not wise to withdraw the inner cannula to remove any possible plug, because this would have left the sharp point of the needle exposed and liable to lacerate the heart wall.

Percussion over the upper cardiac region after the patient had been tapped showed clearness where previously there had been dulness. The woman, after the operation, seemed to breathe more easily, and had a better pulse. She was somewhat excited during the operation, but did not seem to suffer much real pain. Ethyl chloride was used to freeze the skin before the puncture was made.

Before the operation there was dulness of the right chest posteriorly, with moist rales. There was marked dulness over the heart. The dull area was somewhat irregularly triangular, with the base downward. This dulness extended into the left axillary region. The dull area seemed to be displaced forward when the patient turned on the right side. The cardiac sounds and impulse were quite distinct in front, and a friction sound was heard at the base of the heart. This was said to be less than on the previous day. The cardiac sounds were heard posteriorly. There were some rales posteriorly in the left chest. The patient was breathing very rapidly, and had a weak, frequent pulse, but was able to lie flat on 
her back even before operation. The day after operation she was in better condition than on the day of operation, and the cardiac dulness, which had been diminished by operation, was not increased.

On March 12, 1904, at 12.30 P.M., it was determined to make another aspiration, because the pericardial sac had evidently refilled. A puncture was made with the Roberts trocar in the fifth interspace, two and three-quarter inches to the left of the middle line of the sternum. The aspirator pump was attached, but nothing was obtained, except a very few drops of bloody fluid. The trocar was then withdrawn and found to be plugged. A second puncture was then made in the same interspace, three and threequarter inches from the mid-line of the sternum. The puncture, made a few days previously, had been made, in the interspace above, three and a half inches from the mid-line of the sternum and through the lower portion of the breast gland. 'The second puncture, made to-day, obtained by aspiration four and one-half fluidounces of dark-bloody fluid. The fluid was much darker than that obtained a few days ago, and did not coagulate so quickly as that did, if it coagulated at all. It was thicker than the previously obtained fluid. The trocar moved with the heart beats in both punctures to-day, but not so vigorously as it did on the former occasion. At the last puncture the trocar was buried in the tissues about $9 \mathrm{~cm}$. The hand of the operator received the impression that the point was in a large cavity. At the first puncture the end seemed not to enter a large cavity, but to strike against some solid body within the chest. An increased resonance was obtained by percussion of the precordial region after the operation. The patient died early the next(?) morning.

The autopsy showed the pericardial cavity nearly free from fluid; the heart was covered with shaggy lymph, particularly about its base, and there were adhesions between the heart and pericardium. The punctures in the chest wall were in the fourth and fifth interspaces, and the first puncture made on the second day scraped or went through the edge of the costal cartilage above the space.

Upon the front of the heart was a small ecchymosis without apparent wound that looked as if it might have been caused by contact with the end of the trocar. This fact could not be definitely confirmed, as the point of ecchymosis was not very marked. There was a considerable amount of fluid in the left pleural cavity, and a condition of pleuropneumonia. There was some congestion and consolidation of the right lung.

CASE II.-Colored man, aged thirty-one years, in the Polyclinic Hospital under Dr. Riesman. Adinitted on March 22, 1904; died on March 28, 1904.

On March 24, 1904, at 11 P.M., I found an enlarged area of cardiac dulness, and valvular murmurs present and easily heard. The 
apex beat was seen and felt under the left nipple. I did not believe there was much fluid in the pericardium, though the dulness extended to the right of the sternum. Heart was supposcd to be enlarged. Some dulness of the right chest existed posteriorly, with some harsh breathing. At the right base posteriorly there was heard what seemed like pleural friction, but I was uncertain whether it was not rales. Left chest posteriorly showed some dulness. The question in my mind was whether the pulmonary congestion was secondary to the heart lesion, or whether the old cardiac valvular disease had the pulmonary condition added to it and was causing weak pulse (130?), rapid respiration, and sweating, and dyspnrea. The man was not sitting up to breathe. I decided that there was not enough fluid in the pericardium to justify tapping. I had not the benefit of Dr. Riesman's presence to aid me in the diagnosis.

Dr. Riesman sent word on March 25th that he thought tapping was desirable, but could not stay to the operation. I saw the patient and found the condition to be about as the day before, but more dulness in the cardiac area appeared to be present. I tapped in the fourth right interspace close to the sternum with Roberts' aspirating trocar, not attached to vacuum until after insertion. Three fluidrachms of slightly reddish fluid were obtained. The trocar did not move with the heart, but with respiration.

I then punctured in the fifth left interspace directly over the apex beat about one and one-half inches below the nipple, as it seemed desirable to be sure there was no large effusion overlooked. The end of the trocar seemed to strike the heart and perhaps to be embedded in the heart muscle, because it moved with such vigor with the heart beats. No fluid, except a few drops of blood, escaped. The pump brought very little fluid, and, after repeated attempts to get fluid, the instrument was withdrawn. Atteinpts were made to obtain fluid by altering the position of the end of the cannula, but none was obtained. The end did not feel to be frce in a cavity of any size. The end of the cannula contained blood clot, and there was a free flow of blood from the wound until it was closed with cotton and collodion. The patient at one time complained of sevcre pain, and it was then that the operator felt that the heart had been wounded.

The next day the patient seemed better; the pulse was stronger, and it was thought that the area of cardiac dulness on the right of the sternum was less.

Patient died on Marcl 28th. Autopsy showed no pleural effusion; no fluid in pericardium, but marked amount of lymph in pericardium. The heart was greatly hypertrophied. No mark was found of wound of heart, but this would probably have been concealed by lymph deposits. 